\title{
Mitsuokella multacida
}

National Cancer Institute

\section{Source}

National Cancer Institute. Mitsuokella multacida. NCI Thesaurus. Code C86516.

A species of anaerobic, Gram neg ative, rod shaped bacterium assigned to the phylum Firmicutes. This species is susceptible to Kanamycin and Penicillin, ferments rhamnose, mannitol, D-tagatose and melezitose, grows in the presence of Brilliant green and reduces nitrate. $\mathrm{M}$. multacida is a commensal organism of the gastrointestinal tract. 